\title{
Mindfulness and Divergent Thinking: The Value of Heart Rate Variability as an Objective Manipulation Check
}

\author{
Julia Keller ${ }^{1, *}$, Eric Ruthruff ${ }^{2}$, Patrick Keller ${ }^{1}$ \\ ${ }^{1}$ Department of Teaching, Learning, and Leadership, South Dakota State University, United States \\ ${ }^{2}$ Department of Psychology, University of New Mexico, United States
}

Copyright $\bigcirc 2017$ by authors, all rights reserved. Authors agree that this article remains permanently open access under the terms of the Creative Commons Attribution License 4.0 International License

\begin{abstract}
Mindfulness (MF) is a form of mental training that has been linked to increased creativity in problem-solving. Most MF studies recruit participants interested in meditation, and thus are biased towards positive effects. Participants in this study $(n=73)$ therefore signed up for "mental training," not "mindfulness meditation"; the vast majority (54) had little to no prior meditation experience. This is also the first such experiment to use an objective measure of MF (coherence, a physiological correlate of MF that can be indexed by heart rate variability or HRV) as a manipulation check. Participants were randomly assigned to either MF training or a control condition (a memory exercise), and completed a test of divergent thinking (Unusual Uses Task) before and after mental training. Coherence was significantly higher in the MF group and heart rate was significantly lower in the MF group.
\end{abstract}

Keywords Mindfulness, Heart Rate Variability, Problem-solving, Creativity, Coherence

\section{Introduction}

Mindfulness (MF) is a well-established technique for developing the ability to self-regulate attention and emotion. Specifically, MF can be defined as the cognitive ability to pay attention to the present moment without judgment or attachment to a desired outcome. Studies in the field of health psychology have consistently demonstrated benefits of $\mathrm{MF}$, including reduced pain and stress, improved cognitive functioning, increased positive emotion, and increased ability to inhibit emotionally charged but irrelevant information $[1,2,3,4]$. There is also evidence that MF enhances the ability to sustain attention, shift focus from one object to another, and inhibit undesired elaborative verbal-conceptual processing, thus reducing automatic, habitual patterns of responding [5].

Decreasing the influence of automatic, verbal-conceptual processes on the interpretation of experience through $\mathrm{MF}$ meditation may enhance problem-solving [6]. Ostafin and Kassman [6] hypothesized that MF training would increase the ability to solve insight problems but have no effect on solving noninsight problems. Noninsight problems rely on past experiences and the use of logic (e.g., "To the left of a queen there is a jack, to the right of a king there is a spade . . . Assign the proper suit to each card" [7, p. 183]). Insight problems require the inhibition of habitual responding and the ability to transcend traditional methods for solving problems (e.g., "Show how you can make the triangle below point downward by moving only three of the circles" [7, p. 182]). The reliance on past experience to solve an insight problem often leads to an impasse - an inability to solve the problem.

Ostafin and Kassman [6] randomly assigned 71 undergraduates to a MF or a control condition. MF participants listened to a 10-minute tape with $\mathrm{MF}$ instructions. Control participants listened to a natural history text. Then both groups solved two noninsight problems and three insight problems (each problem was worth one point; participants were given 5 minutes to solve each problem). The MF group demonstrated better performance than the controls on the insight problems $(\mathrm{MF}=1.14, \mathrm{C}=0.75$, $p=.03$ ), but not on the noninsight problems.

The results of this study suggest that a brief MF induction released participants from automatic verbal-conceptual processes, thereby facilitating the ability to solve problems requiring novel responses. The authors claim that these are the first findings linking MF and creativity. However, note that the problems used in their study were insight problems-ones that necessitate convergent thinking, a process of identifying a specific or "correct" target solution - and not creative problems, or ones that require divergent thinking, a process that allows for the generation of multiple ideas when there are no right or wrong answers [8, 9].

\subsection{Colzato et al.'s Study on Mindfulness and Creativity}

One creative problem-solving task that involves divergent 
thinking is the Alternate Uses Task (AUT) [10], in which participants list unusual uses for common items like bricks and spoons and are scored on originality (unique responses) and fluency (total number of each participant's responses). Colzato, Ozturk, and Hommel [11] provided an important contribution to the literature on meditation and creativity. They investigated the impact of two types of MF meditation on convergent and divergent thinking tasks (like the AUT) with a group of 19 meditators (average length of previous meditation practice was 2.2 years). One type of meditation was focused attention (FA), in which meditators narrow their focus on to specific objects or sensations (e.g., one's breath); this is also known as concentrative meditation [12]. The other type of meditation was open-monitoring (OM) or mindfulness meditation; it develops a type of attention that is more distributed or diffuse. The intention of OM is to attend moment-by-moment to thoughts, feelings, and sensations arising in the mind without focusing on any particular object. Many meditation techniques (like the one we used in the present study; see Appendix for a transcript of our guided meditation) use a combination of these two types [12].

Colzato et al. [11] predicted that FA meditation, which requires a state of strong top-down cognitive-control, would facilitate performance on convergent thinking tasks that require focus (e.g., the Remote Associates Task [RAT] where participants have to identify a common associate among three unrelated words). In contrast, Colzato et al. reasoned that OM meditation, which calls for weak top-down control, should facilitate performance on divergent thinking tasks (e.g., the AUT) where more distributed control is needed. Participants completed three different 45-minute sessions: FA meditation, OM meditation, and a guided visualization exercise used as a baseline (BA). After each session, participants completed a 5-minute version of the RAT and a 5-minute version of the AUT.

Because AUT coding is key, and our study also used the AUT, a detailed explanation of how Colzato et al. $[10,11]$ coded the AUT follows. Colzato et al. used the AUT in both of their studies on meditation and creativity. But they gave only a brief explanation of their scoring procedure, stating that the AUT was scored by "two independent readers blinded to the experimental conditions" (p. 3) and, in their second study, to also report Cronbach's alpha scores for the AUT scores. Their raters scored responses on four elements: originality, fluency, flexibility, and elaboration.

Originality: Responses given by $2 \%$ to $5 \%$ of the subjects were scored as "somewhat original" (1 point), while responses given by $1 \%$ or less were scored as "original" ( 2 points).

Fluency was "the total of all responses" [11, p. 3]. Colzato et al. did not discuss whether all responses originally given by participants were counted, even if they were not acceptable responses as described by the AUT manual [9]: Acceptable responses must differ from the rest of the participant's responses for the same object and are not common uses. For example, a common use of a newspaper is for reading (not considered acceptable) whereas an alternate use for a newspaper might be to start a fire.

Flexibility was defined by Colzato et al. as "the number of different categories used" [11, p. 3]. Determining what category a response falls into is necessarily a subjective process. For example, are these two responses for paperclip in the same category: "scratch with it" and "write with it"?

\section{Elaboration was:}

the amount of detail, e.g., 'a doorstop' counts 0 , whereas 'a door stop to prevent a door slamming shut in a strong wind' counts 2 (1 point for introducing door slamming and another for providing further detail about the wind) (p. 3).

Although more detail was provided with this description, it seems difficult to decide how many points to give a response like this for paperclip: "poke holes in certain objects". There are more words included than in the basic response of "poke holes," but does the phrase "in certain objects" provide an additional level of detail about the use?

As predicted, participants' scores in Colzato et al. [11] were higher on the divergent thinking task (the AUT) after the $\mathrm{OM}$ session in originality $(\mathrm{FA}=0.8, \mathrm{OM}=2.0, \mathrm{BA}=0.4$, $p<.001)$ and fluency $(\mathrm{FA}=19.3, \mathrm{OM}=24.4, \mathrm{BA}=0.4$, $p<.001$ ). Scores on the convergent thinking task (the RAT) were not significantly better in the FA session than the OM and BA sessions.

The results of Colzato et al.'s study [11] provide new and interesting avenues for future researchers to explore. However, since all of the participants were meditators, self-selection and confirmation bias cannot be ruled out. Participants who sign up for mediation studies may exhibit two types of bias. The first type is known as confirmation bias - a tendency for participants to look for positive affect that confirms their preconceptions about the benefits of meditation (e.g., that meditation creates a state of relaxation). Common wisdom associates relaxation with greater creativity (e.g., you do your best thinking in the shower [13]). So participants who anticipate that meditating will lead to a relaxed state of creative flow [14] may be motivated to try harder to produce more creative responses than a participant who has no such expectations.

A second type of bias that arises when interpreting evidence from MF research is self-selection bias. Do people who have practiced meditation or who sign up for meditation studies have different characteristics than those who choose not to practice meditation? For example, people who have a predisposition for sustained concentration and introspection might naturally choose to meditate, and this may be the reason for their superior cognitive skills. They may also prefer a meditative state over a baseline state (as in Colzato et al.'s study [11]) and this too might account for differences between meditative sessions and baseline. To be able to disentangle training effects from self-selection bias, it is therefore necessary to conduct studies that include novice meditators and participants who are not necessarily 
interested in meditation in order to fully investigate the impact of training on neural and cognitive systems [15].

\subsection{The Present Study}

To reduce the effect of these biases, participants in the present study $(n=73)$ signed up for "mental training," not "mindfulness meditation." In contrast to Colzato et al.'s [11] study, where all the participants had previous experience with meditation, 54 of our participants had little to no prior meditation experience. Use of the neutral language ("mental training") in our recruitment ad may have led to a more balanced sample than in other studies where the words "meditation" or "mindfulness" were used in recruitment.

Ours is also the first such experiment to use an objective measure of MF (heart rate variability, HRV) as a manipulation check. A physiological correlate of MF is coherence, a state of relaxed alertness that enhances emotional stability and cognitive performance [17] that can be indexed by HRV. Having an objective manipulation check is important because: (a) when there are no effects on the dependent variable (in this case, creativity), the researcher knows it is not due merely to manipulation failure; (b) one can increase sensitivity to detect MF effects by eliminating non-adhering or resistant participants; (c) when MF effects are found, one can then further show that it is strongest in the responders (i.e., a stronger 'dose' leads to a larger effect). More generally, the existing survey measures of the degree of MF are very subjective and somewhat controversial [16]. So, having an objective measure of MF might pave the way to a better understanding of MF and how it affects the nervous system. See discussion section for more on the importance of having an objective MF manipulation check.
Coherence is an indication of balance between the sympathetic and parasympathetic nervous systems. The Institute of HeartMath has developed biofeedback technology that teaches individuals how to self-induce a state of psychophysical coherence- - "a highly efficient functional mode associated with increased nervous system harmony, enhanced emotional stability, and improved cognitive performance" [17, p. 130]. Interactive hardware/software monitors and displays HRV patterns as individuals practice breathing mindfully. Heart rhythm patterns become smoother and more sine-wave-like as the practitioner's state of coherence increases (see Figure 1).

The Heartmath software analyzes and records heart rhythm patterns and calculates a coherence ratio for each session. Stable and regular oscillations in heart rhythm frequencies describe high coherence. Low coherence "is characterized by a lower frequency, more disordered heart rhythm pattern and increasing mean heart rate" [18, p. 12].

Is it possible to significantly increase a person's state of coherence after a single, brief session of MF practice, even if that person has never meditated before and has not self-selected to participate in a meditation study? Answering this question could have many useful applications, because coherence improves attentional focus [17], which is critical for cognition. Any improvements in real-world cognitive performance (e.g., while test-taking, responding to crises, making critical decisions) would be highly beneficial.

To summarize, our study sought to answer these questions: Does MF practice improve creative problem-solving on a divergent thinking task? Will meditation-naïve participants, who are not necessarily interested in or predisposed toward practicing $\mathrm{MF}$, be able to enter a meditative state of coherence? Can MF practice increase coherence?

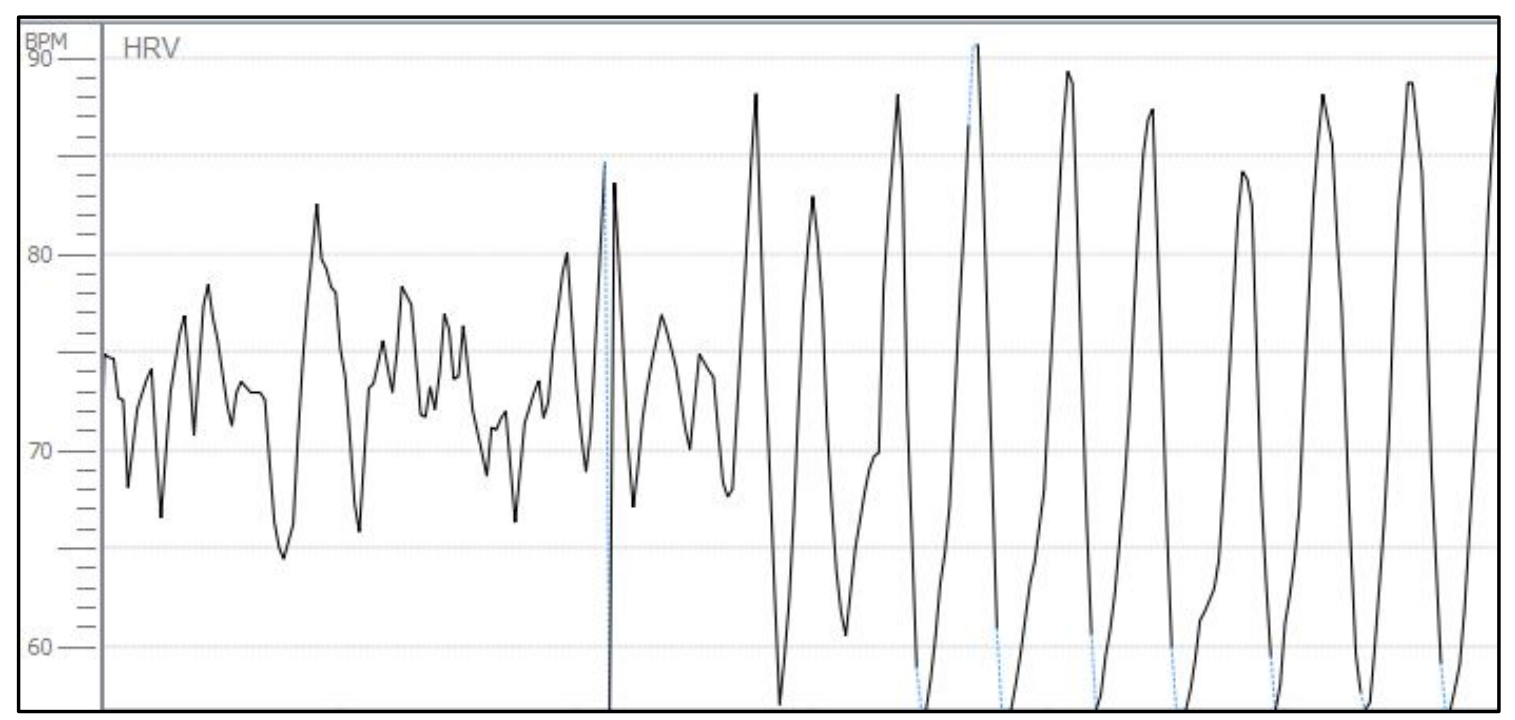

Figure 1. Example of a Heart Rate Variability pattern becoming more coherent over time 


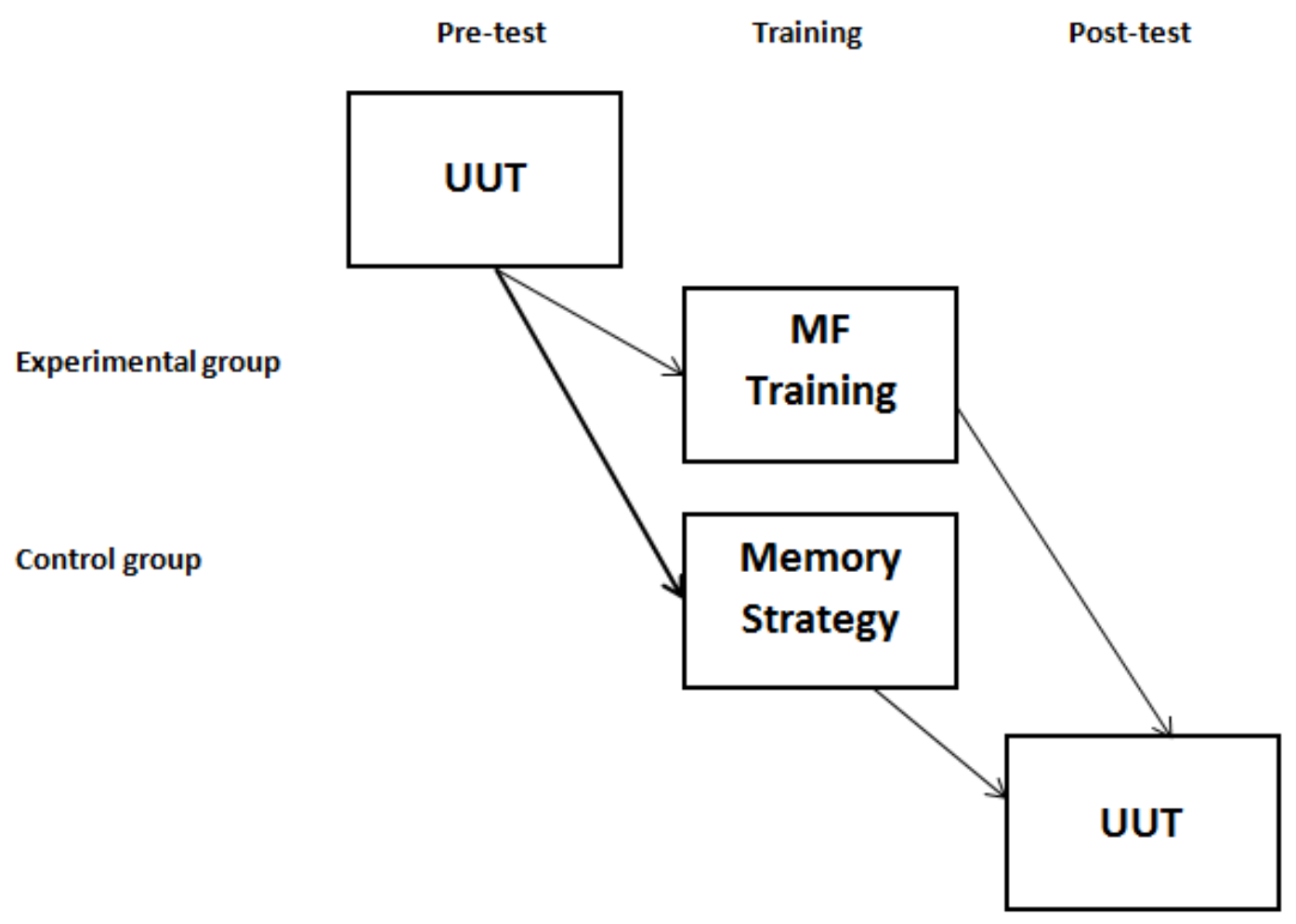

Figure 2. Timeline of study events

\section{Methods}

73 University of New Mexico undergraduates $(M$ age $=20)$ volunteered to participate in the study for credit in a psychology course. The message that they read on the web-based recruitment system maintained by the psychology department was as follows:

The purpose of this research project is to learn more about how mental training affects automaticity and executive control. First, you will be a given a set of instructions specifying a task that you will need to perform. Then, you will receive instructions on mental training. When you have finished, you will take another test.

Participants signed consent forms and were randomly assigned to either MF training or a control condition (a memory exercise). Participants in both groups completed a test of divergent thinking (the Alternate Uses Task) before and after mental training (see Figure 2 for a timeline of study events). During instructions for the AUT, participants were told that the experiment was about the relationship between mental training and creativity. Mental training was defined as "training our mind to be more attentive and/or to remember more." Participants were told this so that the group given a memory exercise would not know they were in the control group, thereby possibly affecting their motivation and thus the amount of effort they put into the creativity task. And, because research demonstrates that giving participants instructions to be creative increases validity of scores on divergent thinking measures [19], participants were instructed to be creative and think of unusual uses for objects. They were then given examples of common and unusual uses for a chair.

After listing unusual uses for four common objects (brick, paperclip, newspaper, and spoon; 2 minutes/object), participants in the experimental group listened to 20 minutes of audiotaped MF instruction (see Appendix for a transcript) during mental training, while those in the control group used the method of loci mnemonic to memorize a list of names. After 20 minutes, participants in the control group were given two minutes to write down all the names they could recall.

During mental training in both conditions, HRV data was collected by an ear sensor. Participants were told that "we are interested in your heart rate while you do a simple task." The window displaying their HRV pattern was minimized during the session so that participants could not see it.

After mental training, participants again listed alternate uses for four common objects (brick, paperclip, hat, and shoe).

\subsection{Data Coding}

In our first run of the experiment, two research assistants independently coded responses by each participant on fluency (as defined by the AUT manual) and creativity (on a 5-point Likert scale; $1=$ most common; 5 = most creative). Inter-rater agreement was .72 for fluency and .14 for creativity. There was considerable difference between the raters on determining "creativity" due to multiple and 
equally valid definitions and perspectives on the construct "creativity." For example, this response for spoon_-"make a waterfall in your sink"—was given a "5" (highly creative) by one rater and a "0" (not an acceptable use) by the other.

So we decided to only use Colzato et al.'s [11] fluency and originality categories, since they were the most clearly defined, and train a new set of coders on consensus coding in order to increase reliability and reduce subjectivity and bias. Consensus coding was a collaborative process where raters (blind to condition) first rated responses on fluency and originality and then met to compare responses and come to a consensus on points of disagreement [20,21]

\section{Results}

Biometric data (coherence and heart rate) and two measures of creativity (fluency and originality scores from the AUT) were collected from each participant. $T$-tests were conducted on post AUT scores and on coherence and heart rate scores with group (experimental vs. control) as a between-subjects factor.

\subsection{Biometric Results: Coherence and Heart Rate}

Coherence is calculated by the HeartMath program in the following manner [18]:

Heart rhythm coherence is reflected in the HRV power spectrum as a large increase in power in the low frequency (LF) band (typically around $0.1 \mathrm{~Hz}$ ) and a decrease in the power in the very low frequency (VLF) and high frequency (HF) bands. A coherent heart rhythm can therefore be defined as a relatively harmonic (sine-wave-like) signal with a very narrow, high-amplitude peak in the LF region of the HRV power spectrum and no major peaks in the VLF or HF regions. Coherence thus approximates the LF/ (VLF + HF) ratio...First, the maximum peak is identified in the 0.04-0.26 Hz range (the frequency range within which coherence and entrainment can occur). The peak is then determined by calculating the integral in a window $0.030 \mathrm{~Hz}$ wide, centered on the highest peak in that region. The total power of the entire spectrum is then calculated. The coherence ratio is formulated as: (Peak Power/(Total Power- Peak Power) ${ }^{2}$. This method provides an accurate measure of coherence that allows for the nonlinear nature of the HRV waveform over time. (p. 8)

After each session, the Heartmath software recorded a total coherence score (ranging from 0-16), a low coherence score (percentage of time with no sine-wave-like activity in the HRV wave), a medium coherence score (percentage of time of some wave-like activity), a high coherence score (percentage of time of very sine-wave-like activity), and average heart rate.

The MF group had significantly higher overall coherence $(\mathrm{MF}=1.13, \mathrm{C}=0.74, \mathrm{t}(72)=-3.44, p<.001)$ and lower heart rates $(\mathrm{MF}=73, \mathrm{C}=79, \mathrm{t}(72)=2.31, p<.02)$ than the control group. (See Figures 3 and 4 for high coherence and heart rate results for novice and experienced participants by condition.)

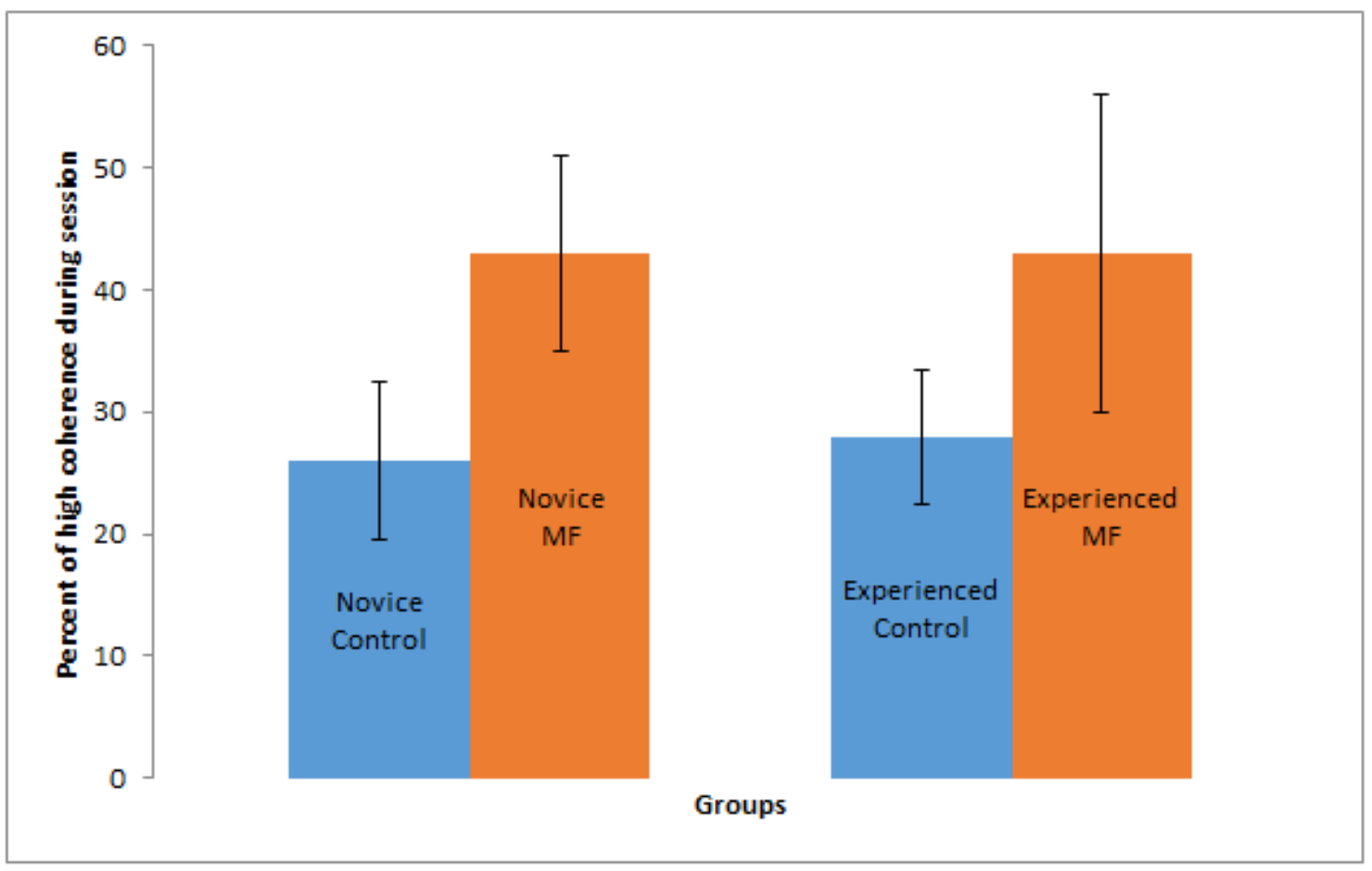

Figure 3. High coherence results. "Novice Control" and "Novice MF" were participants in those groups who had little to no prior meditation experience. "Experienced Control" and "Experienced MF" were participants in those groups who had more experience with meditation. 


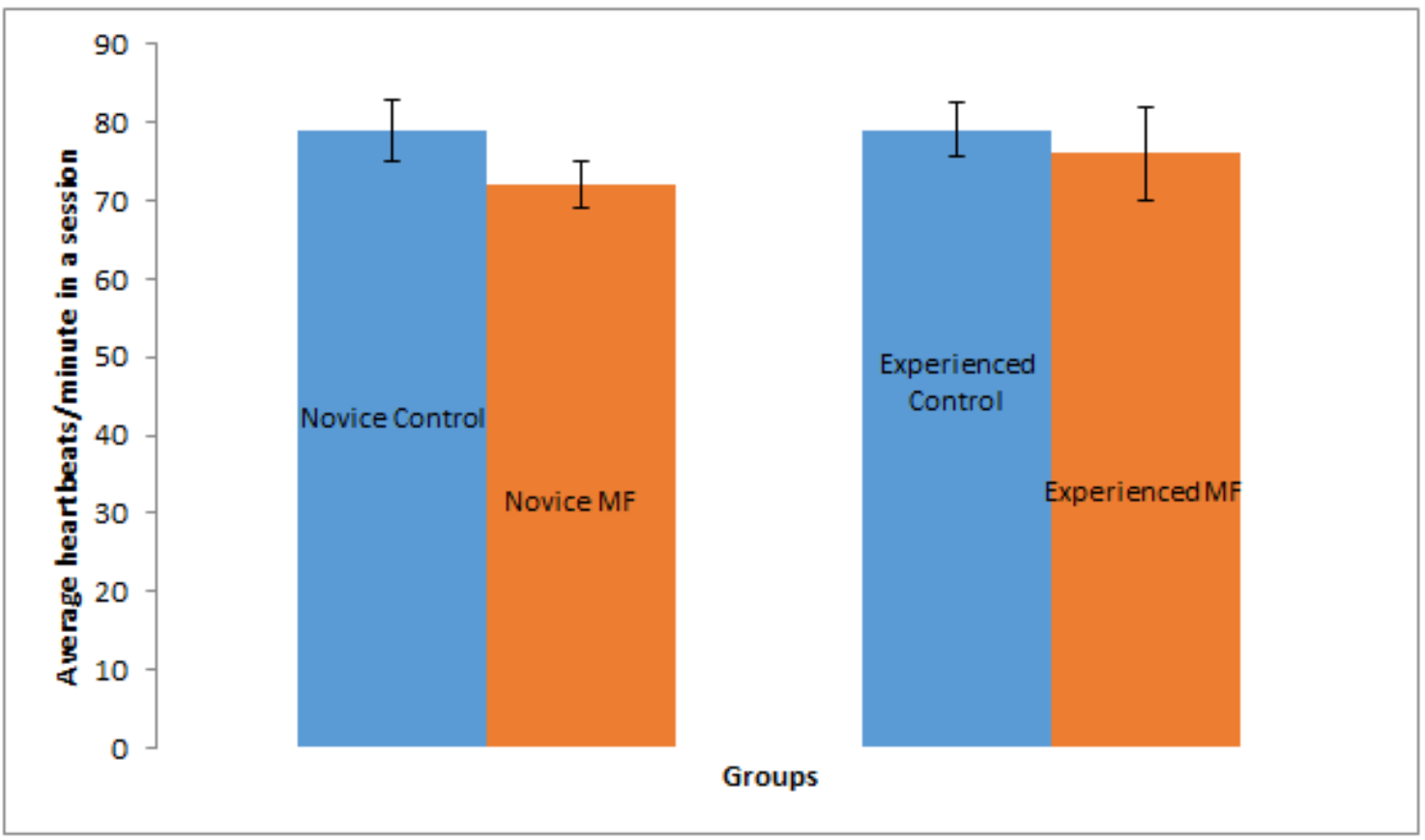

Figure 4. Heart rate results

Eliminating the experienced group scores, the novice MF group still had significantly higher overall coherence (Novice $\mathrm{MF}=1.11$, Novice $\mathrm{C}=0.72, \mathrm{t}(54)=-3.11, p<.002)$ and lower heart rates (Novice $\mathrm{MF}=72$, Novice $\mathrm{C}=78, \mathrm{t}(54)=2.15$, $p<.02)$. There were no significant differences between the experienced and novice groups in age or sex.

\subsection{Creativity Scores}

There was no significant effect of MF on post-test AUT fluency (MF $=15.15,95 \%$ CI $[12.88,17.42]$; $\mathrm{C}=15.74,95 \%$ CI $[13.62,17.86])$ or originality $(\mathrm{MF}=14.31,95 \% \mathrm{CI}[11.92,16.7] ; \mathrm{C}=15.32,95 \% \mathrm{CI}[12.4,18.24]$; see Figures 5 and 6$)$.

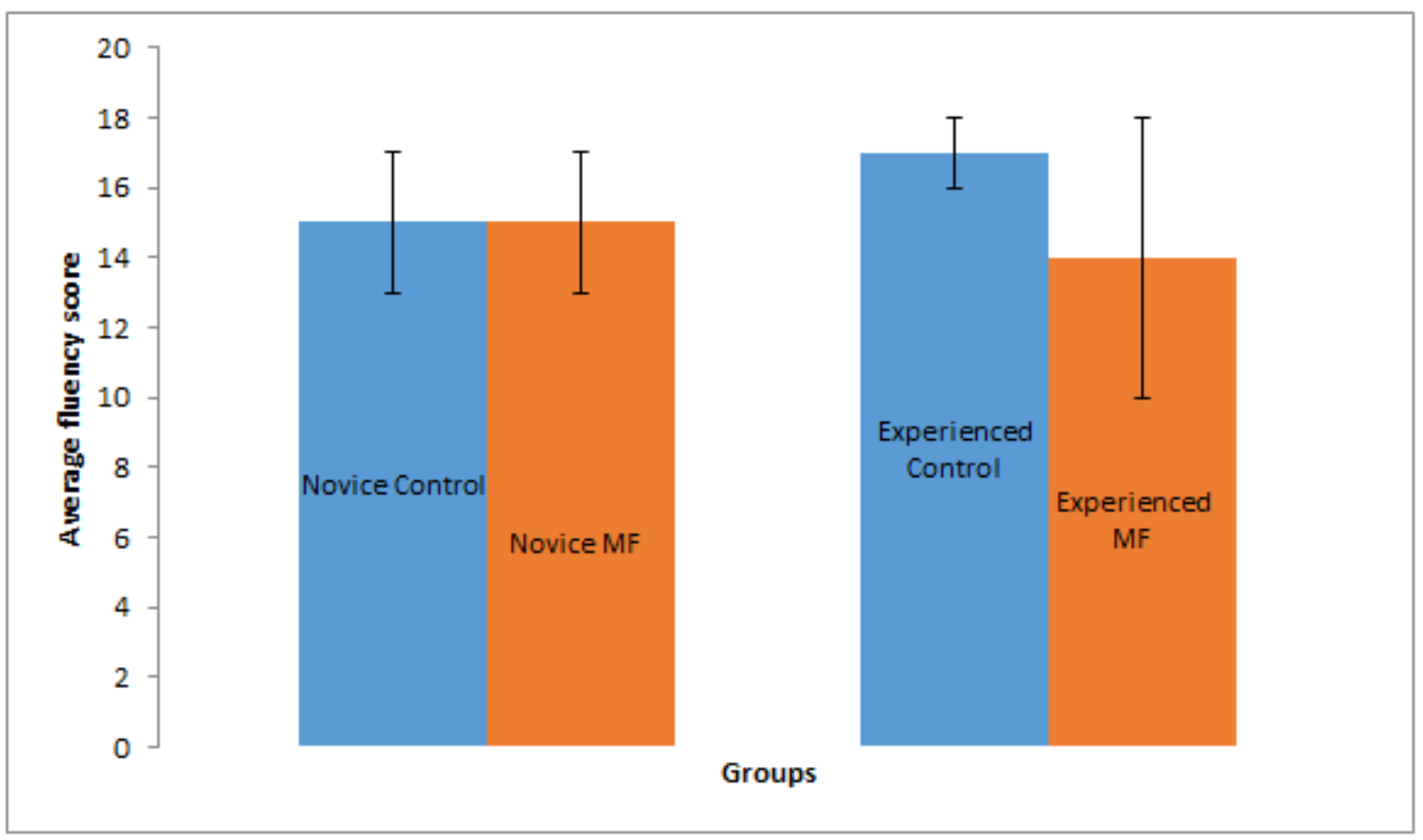

Figure 5. Fluency scores on post-test 


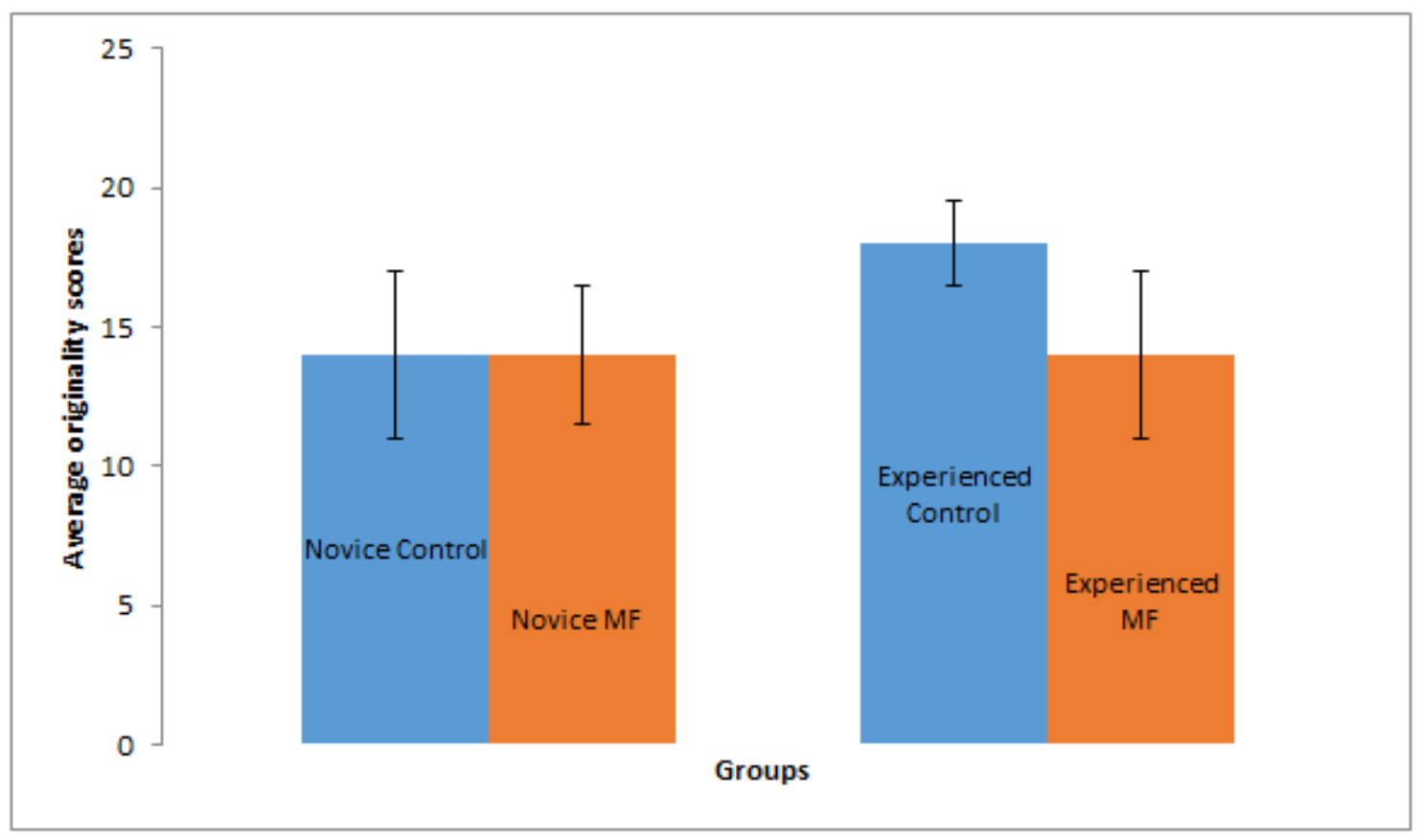

Figure 6. Originality scores on post-test

\section{Discussion}

Heart rate was significantly lower in the MF group and coherence was significantly higher. Both low heart rate and coherence are generally considered beneficial for physical and psychological health [22] and could be especially useful in academic settings where test anxiety has debilitating effects on school performance, intelligence tests, and standardized tests [23]. The perception of a perceived threat (e.g., failing a test) causes the release of adrenaline, which increases heart rate and muscle tension and raises blood pressure. Repeated stimulation of the stress-response system over long periods of time can cause numerous mental and physical problems including anxiety, depression, sleep problems, impairment of memory and concentration, high blood pressure, irregular heart rhythms, digestive problems, and a weakened immune system $[24,25]$. Breathing slowly can reverse some of the adverse effects of stress by increasing oxygen saturation in the cells, alpha waves, and heart rate variability and lowering heart rate, thereby releasing energy and improving cognitive abilities [26].

Nevertheless, despite evidence of increased coherence, MF practice did not significantly improve creative problem-solving on the AUT. Hence, we did not replicate the results of Colzato et al. [11]. This may be due to two factors. First, we used different AUT scoring methods (detailed in the Data Coding section above). A second factor may have been a sample issue. In a follow-up study, Colzato et al. [9] aimed to replicate and extend their previous findings by comparing 20 meditation practitioners with 20 novices (Note: the authors did not describe whether or not novice volunteers knew they were signing up for a meditation study). Whereas practitioners' fluency scores were significantly higher in the $\mathrm{OM}$ condition than in the FA condition $(\mathrm{FA}=4.9 ; \mathrm{OM}=6.4, p<.05)$, novice's fluency scores were not $(\mathrm{FA}=5.6, \mathrm{OM}=6.3)$ and there was no significant effect in either group of meditation type on originality scores. This suggests that long-term meditation experience does significantly increase creative fluency on divergent thinking tasks and this could be another reason our findings did not reach significance-because $74 \%$ of our sample had little to no prior meditation experience. This again suggests that self-selection and confirmation bias may have influenced Colzato et al.'s [11] results (a point acknowledged by the authors themselves).

\subsection{Implications for Future Research}

The present study offers suggestions for future research. Other studies have found that incubation, a period of time when a problem is put aside, enhances the solving of problems that lead to an impasse [8]. Ostafin and Hassman [6] postulated that MF helps insight problem-solving because it reduces automatic verbal-conceptual processing, enabling a greater mental clarity that allows for more freedom in creative response. However, Ostafin and Kassman did not investigate $\mathrm{MF}$ as a potential form of incubation because they did not include an incubation period between problem-solving sessions. MF, because of its release from rumination and other non-productive forms of elaborative processing, might prove to be especially beneficial when solving problems with significant negative emotional valences (e.g., frustration). Our study included an incubation period, and there were participants in both groups who increased in fluency and originality. Future research might examine whether or not MF was more 
effective than other forms of incubation that have also been found to be effective in insight problem-solving (e.g., rest, low-demand tasks, and mind-wandering).

Another suggestion for future research concerns how to score divergent thinking tasks. Scoring of divergent thinking tasks has often been criticized for having low validity and reliability. Silvia et al. [27] gives some reasons why it is difficult to decide whether a response is appropriate or unique or simply bizarre and inappropriate.

Any researcher who has implemented the 0/1 system knows that the line between "creative" and "random" is often fuzzy. Researchers will disagree, for example, over whether "a round cube" or "a roundhouse kick from Chuck Norris!" should be filtered as capricious, inappropriate responses to a "things that are round" task. (p. 69)

We attempted to remedy this problem by employing consensus coding with raters blind to condition. Consensus coding has not been used before with creativity measures such as the AUT and future studies using this method could be conducted to determine whether it offers a more valid and reliable method for scoring subjective measures.

This is also the first experiment to use an objective measure of MF as a manipulation check. A relationship between higher levels of MF and high-frequency HRV (associated with increased activity in the parasympathetic or rest-and-digest component of the autonomic nervous system) has been found in several meditation studies [28, 29]. Wu and Lo [29] found that meditation techniques characterized by slow breathing resulted in regular oscillations (or coherence) in high-frequency HRV. Mankus, Aldao, Kerns, Mayville, and Mennin [30] found that higher levels of MF were related to higher levels of HRV (using a metric that indexed the degree of parasympathetic influence on heart rate) in participants with elevated general anxiety symptoms, who have more difficulty with emotion regulation. They explain this relationship as follows: MF practice may enhance the ability to regulate emotions - thoughts and emotions can be perceived without immediately acting on them-and increased HRV is an autonomic indicator of flexibility in emotion regulation.

Porges' Polyvagal Theory [31] offers an explanation for the connection between emotion regulation and HRV. The tenth cranial nerve is the vagus nerve, which regulates the heart and bronchi. The myelinated vagus acts on the motor neurons of the sinoatrial node (that performs like a pacemaker in the heart) to increase or decrease heart rate. Vagal tone is the influence on the heart when only the parasympathetic nerve fibres-carried in the vagus nerve-are determining heart rate. According to Porges, when vagal tone is high, the vagus limits heart rate, thereby "functionally calming the individual" [31, p. 110]. Thus vagal tone, and its effect on heart rate, is an index of the ability to regulate emotion.

Porges' Polyvagal Theory, and results from studies that have found a close relationship between HRV and MF, indicate that HRV can be used as an objective manipulation check to see whether participants are following MF instructions. This allows researchers to determine the most effective MF manipulations and exclude participants who did not actively engage in MF, thereby improving data quality.

Other MF studies (including ones cited in this paper [4, 6]) have used self-report measures like the Toronto Mindfulness Scales [32] or the Mindful Attention Awareness Scale (MAAS, [33]), or, as in the case of Colzato $[10,11]$, do not measure MF. Self-report scales are considered by many researchers as unreliable indicators of MF. Recent studies in meta-awareness and attention demonstrate that mind wandering (considered to be a mindless state) is related to a lack of meta-awareness (one is aware that they are not aware). These findings indicate that the ability to accurately report about states of mindlessness is limited without training. MF scales like the MAAS contain statements like this: "I do jobs or tasks automatically, without being aware of what I'm doing." Participants then have to rate themselves on how frequently they are mindless. Without MF training, participants are limited in their ability to accurately report mindless states. Lack of meta-awareness concerning mindless states and social desirability bias (trying to answer questions the way they think the experimenter wants them to answer [34]) suggests that responses on the MAAS to statements like this one are inaccurate [35]. Thus, there is a strong need to move from subjective self-report measures of MF to objective ones.

Even though $74 \%$ of our sample had little-to-no experience with meditation and participated in our study for course credit rather than because they were interested in MF, they were able to enter into a meditative state that significantly increased their psychophysiological state of coherence and lowered their heart rate. Future studies on the effect of MF on cognition would benefit from also using HRV as an objective manipulation check in order to establish whether or not participants are following MF instructions.

\section{Appendix}

\section{Mindfulness Meditation Transcript}

In any seated position, you can allow your hands to rest on your thighs, or fold them in front of you. Please feel free to alter your position as needed during the meditation. Allow your back to be erect, with your head balanced on top of your neck and chest, dignified and present. With your head directly above your tailbone, allow all the muscles of your body to soften, as much as they can in this position. Take this time to honor your decision to attend to your mind and body in this fashion. Eyes can be open or closed. If 
open, simply allow your gaze to remain in one place, just in front of you, taking a break from scanning and searching, but remaining open to the sense of sight. Or allow your eyes to close, gently. Calmly bring your attention to the breath, without altering the breath too much. Take a moment to explore the place in your body where breathing is most obvious and natural. The nose, the mouth, the throat, the chest, or the belly. Allow your attention to rest here. Exploring the sounds, the movements, the body sensations, the nourishment, and the cleansing involved in breathing. Bringing attention to the moment between in-breath and out-breath. Following each breath, all the way to the end. Breath after breath. Moment after moment. Just breathing. Mindful. Just sitting. Inhabiting the moment. Dropping into stillness. Just breathing. Sitting.

You may notice that the mind has a tendency to wander from the breath. When this happens, simply observe the mind doing this. Taking note of which thought, feeling, or body sensation arose. Then, gently escort your attention back to breathing. Seeing each breath as a new event. As if you were breathing for the first time. Noticing its waves, its fluid nature, its rhythm. You may notice that, when the mind wanders, it is easy to become critical of oneself for having such a mind. Taking note of this type of judgment, realizing it is the nature of the mind to wander, escort your awareness back to breathing. Simply redirecting the mind back to the present moment, with kindness and without judgment, is the most fundamental aspect of the practice of mindfulness. The breath is like an anchor. This CD has some extended periods of silence. During these silences, you can practice mindfulness. And if your mind wanders, you can always bring awareness back to breathing. Breathing. Mindful. Just sitting. Inhabiting the moment. Dropping into stillness. Just breathing. Sitting.

You may become aware of emotions, which are somehow associated with breathing. Or which may arise as the mind becomes less distracted. These emotions can also be observed in the same way as thoughts and body sensations with kind awareness, just allowing them to be there. Taking note of the range of emotions present. Of whether you like or dislike them. And then calmly redirecting focus to the breath. Breathing. Mindful. Just sitting. Inhabiting the moment. Dropping into stillness. Just breathing. Sitting.

You may notice that although the mind always tends to wander into the past or the future, the actual thoughts, pains, pleasures and sounds to which it wanders are constantly changing. Taking notice of this impermanence of things, allow the mind to return to the constant, ever-present breath. Sitting still, solid as a mountain, while the procession of thoughts, sensations, and feelings can be seen as clouds passing over the mountain. These clouds may swirl around the mountain, unleashing wind and rain, but they will eventually pass. The mountain remains still and solid, co-existing with the clouds, yet not reacting to them. Breathing. Breathing. Mindful. Just sitting. Inhabiting the moment. Dropping into stillness. Just breathing. Sitting.
Coming to the end of the formal meditation, bringing awareness to the present moment. Knowing it is possible to bring awareness at any time of day, allowing yourself to step out of the constant stream of thoughts, feelings, and sensations, if even just for a minute. This constant return to awareness is the practice. Whether sitting here, or washing dishes, or driving a car. There are countless opportunities in daily life to practice being attentive to the acts of clinging to things we like, and resisting things we don't like. By focusing on the breath or the body in the present moment, the mind can settle and perceive things, how they really are. Breathing. (Bell: 3 times)

\section{REFERENCES}

[1] Y. Tang, M.I. Posner, M. I. Attention training and attention state training. Trends in Cognitive Sciences, Vol. 13, No. 5, 222-227, 2009.

[2] R.J. Davidson, J. Kabat-Zinn, J. Schumacher, M. Rosenkranz, D. Muller, S.F. Santorelli, F. Urbanowski, A. Harrington, K. Bonus, J.F., Sheridan. Alterations in brain and immune function produced by mindfulness meditation. Psychosomatic Medicine, Vol. 65, 564-570, 2003.

[3] M. Majumdar, P. Grossman, B. Dietz-Waschkowski, S. Kersig, S.H. Walach, H. Does mindfulness meditation contribute to health? Outcome evaluation of a German sample. Journal of Alternative and Complementary Medicine, Vol. 8, No. 6, 719, 2002.

[4] C.M.M. Ortner, S.J. Kilner, P.D. Zelazo, P.D. Mindfulness meditation and reduced emotional interference on a cognitive task. Motivation and Emotion, Vol. 31, 271-283, 2007.

[5] S.R. Bishop, M. Lau, S. Shapiro, L. Carlson, N.D. Anderson, J. Carmody, Z.V. Segal, S. Abbey, M. Speca, D. Velting, G.D. Devins. Mindfulness: A proposed operational definition. Clinical Psychology: Science and Practice, Vol. 11, No. 3, 230-241, 2004.

[6] B.D. Ostafin, Kassman, K. T. Stepping out of history: Mindfulness improves insight problem solving. Consciousness and Cognition, Vol. 21, 1031-1036, 2012.

[7] J.W. Schooler, S. Ohlsson, K. Brooks. Thoughts beyond words: When language overshadows insight. Journal of Experimental Psychology, Vol. 122, No. 2, 166-183, 1993.

[8] U.N. Sio, U. N., T.C. Ormerod. Does incubation enhance problem-solving? A meta-analytic review. Psychological Bulletin, Vol. 135, No. 1, 94-120, 2009.

[9] L.S. Colzato, A. Szapora, D. Lippelt, B. Hommel. Prior meditation practice modulates performance and strategy use in convergent- and divergent-thinking problems. Mindfulness, 1-7, 2014.

[10] J.P. Guilford, P.R. Christensen, P.R. Merrifield, R.C. Wilson. Alternate uses: Manual of instructions and interpretation. Orange, CA: Sheridan, 1978. 
[11] L.S. Colzato, A. Ozturk, B. Hommel. Meditate to create: the impact of focused-attention and open-monitoring on convergent and divergent thinking. Frontiers in Psychology, Vol. 3, 1-5, 2012.

[12] B.R. Cahn, J. Polich. Meditation states and traits: EEG, ERP, and neuroimaging studies. Psychological Bulletin, Vol. 132, No. 2, 180-211, 2006.

[13] Stop trying! Creativity's ultimate partner is relaxation. Online available from http://www.brainsync.com/blog/stop-trying-creativitys-ultim ate-partner-is-relaxation/

[14] M. Czikszentmihalyi. Creativity: Flow and the psychology of discovery and invention. New York: Harper, 1996.

[15] R.J. Davidson. Empirical explorations of mindfulness: Conceptual and methodological conundrums. Emotion, Vol. 10, No. 1, 8-11, 2010.

[16] K. Dyga, R. Stupak. Meditation and psychosis: Trigger or cure? Archives of Psychiatry and Psychotherapy, Vol. 3, 48-58, 2015.

[17] R. McCraty, R. Enhancing emotional, social, and academic learning with heart rhythm coherence feedback. Biofeedback Vol. 33, No. 5, 130-134, 2005. Online available from http://humancair.com

[18] R. McCraty, M. Atkinson, D. Tomasino, R.T. Bradley. The coherent heart: Heart-brain interactions, psychophysiological coherence, and the emergence of a system-wide order, 2006. Online available from

http://www.HeartMath.com/wp-content/uploads/2014/04 /coherent heart.pdf

[19] P.J. Silvia. Subjective scoring of divergent thinking: Examining the reliability of unusual uses, instances, and consequences tasks. Thinking Skills and Creativity, Vol. 6, 24-30, 2011.

[20] J. Saldaňa. The coding manual for qualitative researchers. Los Angeles, CA: Sage, 2013.

[21] D.G. Hays, A.A. Singh, (2012). Qualitative inquiry in clinical and educational settings. New York: Guilford Press, 2012.

[22] J.M. Burg, O.T. Wolf, O. T., J. Michalak, J. Mindfulness as self-regulated attention: Associations with heart rate variability. Swiss Journal of Psychology, Vol. 71, No. 3, 135-139, 2012.

[23] S. Swanson, C. Howell. Test anxiety in adolescents with learning disabilities and behavior disorders. Exceptional Children, Vol. 62, No. 5, 389-397, 1996.
[24] Cleveland Clinic. Stress and heart disease, 2013. Online available from http://my.clevelandclinic.org/services/heart /prevention/emotional-health/stress-relaxation/stress-manage ment -your-heart

[25] Mayo Clinic. Chronic stress puts your health at risk, 2013. Online available from http://www.mayoclinic.com/health/stress /SR00001

[26] C. Thompson. You're breathing all wrong. Men's Journal, 2009. Online available from http://www.mensjournal.com/magazine/you-re-breathing-allwrong-20130227

[27] P.J. Silvia, J.T. Winterstein, C.M. Barona, J.T. Cram, K.I. Hess, J.L. Martinez, C.A. Richard. Assessing creativity with divergent thinking tasks: Exploring the reliability and validity of new subjective scoring methods. Psychology of Aesthetics, Creativity, and the Arts, Vol. 2, No. 2, 68-85, 2008 .

[28] Y.Y. Tang, Y. Ma, J. Wang, Y. Fan, H. Feng, J. Wang, A. Feng, Q. Lu, B. Hu, Y. Lin, J. Li, Y. Zhang, Y. Wang, L. Zhou, M. Fan. Central and autonomic nervous system interaction is altered by short-term meditation. Proceedings of the National Academy of Sciences of the United States of America, Vol. 106, 8865-8870, 2009.

[29] S. Wu, P.C. Lo. Inward-attention meditation increases parasympathetic activity: A study based on heart rate variability. Biomedical Research, Vol. 29, No. 5, 245-250, 2008.

[30] A.M. Mankus, A. Aldao, C. Kerns, E.W. Mayville, D.S. Mennin. Mindfulness and heart rate variability in individuals with high and low generalized anxiety symptoms. Behaviour Research and Therapy, Vol. 51, No. 7, 386-391, 2013.

[31] S.W. Porges, S.A. Furman. The early development of the autonomic nervous system provides a neural platform for social behavior: A polyvagal perspective. Infant and Child Development, Vol. 20, 106-118, 2011.

[32] M.A. Lau, S.R. Bishop, Z.V. Segal, T. Buis, N.D. Anderson, L. Carlson, S. Shapiro, J. Carmody, S. Abbey, G. Devins. The Toronto Mindfulness Scale: Development and validation. Journal of Clinical Psychology, Vol. 62, No. 12, 1445-1467, 2006.

[33] K.W. Brown, R.M. Richard. The benefits of being present: Mindfulness and its role in psychological well-being. Journal of Personality and Social Psychology, Vol. 84, No. 4, 822-848, 2003.

[34] P.C. Cozby. Methods in behavioral research (10th ed.). Boston, MA: McGraw-Hill, 2009.

[35] N.T. Van Dam, M. Earleywine, A. Borders. Measuring mindfulness? An Item Response Theory analysis of the Mindful Attention Awareness Scale. Personality and Individual Differences, Vol. 49, 805-810, 2010. 